\title{
CODS-COMAD 2018
}

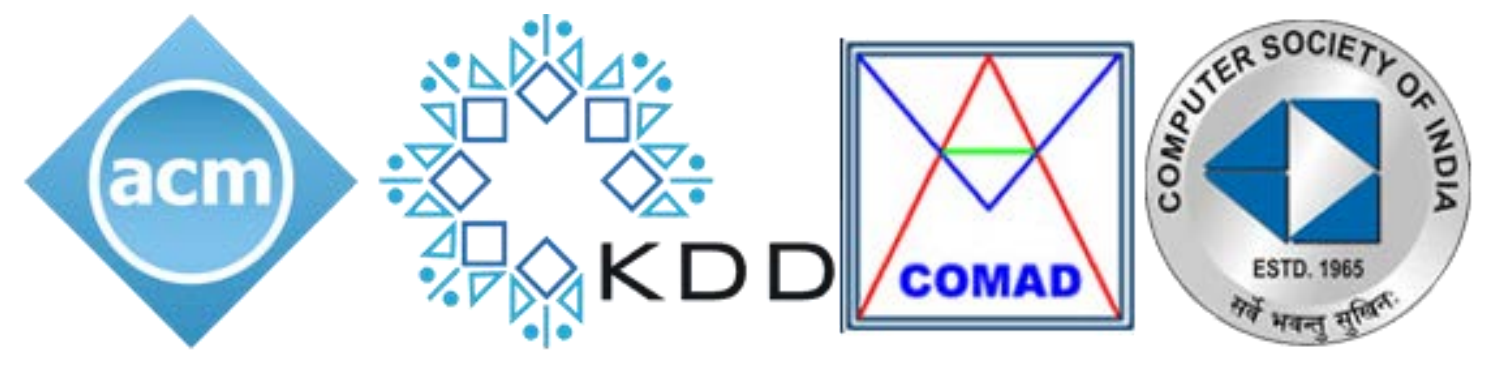

Proceedings of the 5th ACM IKDD CoDS and 23rd COMAD, January 11-13, 2018

Goa, India

Sunita Sarawagi Shourya Roy Program Chairs

Sayan Ranu Proceedings Chair 



\section{Preface}

This volume contains the papers presented at the ACM India Joint International Conference on Data Science and Management of Data (CoDS-COMAD) 2018 (5th ACM IKDD CoDS and 23rd COMAD), Goa, India on January 11 - 13, 2018.

For over two decades, the International Conference on Management of Data (COMAD), modeled along the lines of ACM SIGMOD, has been the premier international database conference hosted in India by Division II of Computer Society of India (CSI). The first COMAD was held in 1989, and it has been held on a nearly annual basis since then. COMAD has always had a significant international participation, with about $30 \%$ of the papers being from outside India, including Europe, USA and East/South-East Asia.

Conference on Data Sciences (CoDS) encompasses the areas of data analytics, machine learning and managing big data hosted by the India Chapter of ACM KDD (IKDD). Since its inception in 2013, CoDS has been successful in putting together a very exciting program in the growing field of Data Science. The series of CoDS conferences have also hosted numerous esteemed speakers from various parts of the world.

The CoDS-COMAD 2018 conference builds on the long history of both COMAD and CoDS towards bringing together the two related communities closer under a common umbrella. This year the joint conference proved to be a big success attracting a large number of registrations. The keynote talks this year are by Anima Anandkumar (Principal scientist at Amazon Web Services \& Endowed Professor at Caltech), Surajit Chaudhuri (Distinguished Scientist, Microsoft Research, Redmond), and Manik Varma (Principal Researcher, Microsoft Research India \& Adjunct Professor of Computer Science, IIT Delhi). There are four tutorials (including two invited ones) covering interesting and relevant topics of data science and databases. As a new initiative, this year's conference also hosts eight invited talks by top researchers from India and abroad. The goal of this new initiative is to make CoDS-COMAD as the meeting place of top researchers in the country and showcase their research to students, industry, and foster future collaboration.

The main research track of CoDS-COMAD 2018 received about 100 submissions of which 30 papers were selected for oral presentations. All selected papers are also presented as posters for additional interactions between authors and audiences. In addition, Industry and Demo tracks as well as the Young Researchers' Symposium accepted about 25 papers through thorough peer review processes. Both the industry track and the Young Researchers' Symposium hosted a mix of invited talks, submitted papers as well an interesting panel discussion such as on the topic "Deep Learning: Hype versus Reality".

It is our pleasure to record our appreciation and gratitude for the invaluable guidance we received from the General Chairs --- Niloy Ganguly, IIT Kharagpur and Raghu Ramakrishnan, Microsoft. Niloy, in particular, has been instrumentally involved throughout the organization and preparation of the conference over the last several months.

Thanks go to the organization committee members, the local organizers and the volunteers without whom it is not possible to organize a conference of this dimension. Special thanks to Venkatesh Kamat, Organizing Committee Chair, for a flawless local organization of the conference. 
It also gives us great pleasure to thank our corporate sponsors, who along with the hosts and ACM India facilitated this conference. Our sincere thanks to all the invited speakers in the conference for agreeing to be a part of CoDS-COMAD 2017.

The success of any conference is ultimately due to the authors who submit papers and the attendees who participate in the proceedings. Our immense thanks are to the authors and conference participants. With such a strong participation from academia and industry, we are sure that the participants will get inspired and create further synergies between academic and industrial researchers and lead to numerous innovations.

Goa, India

Sunita Sarawagi

Shourya Roy

Program Chairs

Sayan Ranu

Proceedings Chair 


\section{Organizing Committee}

General Chairs

Niloy Ganguly, IIT Kharagpur

Raghu Ramakrishnan, Microsoft

\section{Program Chairs}

Sunita Sarawagi, IIT Bombay

Shourya Roy, American Express Big Data Labs

\section{Organizing Chairs}

Venkatesh V. Kamat, Goa University

Vandana Naik, Goa University

\section{Tutorial Chairs}

Arnab Bhattacharya, IIT Kanpur

Vaibhav Rajan, NUS, Singapore

\section{Demo Chairs}

Mitesh Khapra, IIT Madras

Moumita Sinha, Adobe Research

Proceedings Chair

Sayan Ranu, IIT Delhi

\section{Industry Track Chairs}

Prasad Deshpande,Kena Labs

Mayur Datar, Flipkart

\section{Publicity Chairs}

Raj Sharma, Videoken

Mahashweta Das, Visa Research

Finance Chair

Chandrashekhar Sahasrabuddhe, PSPL

\section{Sponsorship Chair}

Balaraman Ravindran, IIT Madras

\section{Graduate Workshop Chairs}

Pawan Goyal, IIT Kharagpur

Amit Awekar, IIT Guwahati

\section{Website Chairs}

Amrith Krishna, IIT Kharagpur

Bhuthesh R, Indian Institute of Science 


\section{Program Committee}

- Aditya Telang, American Express

- Akshar Kaul, IBM Research

- Amitabha Bagchi, IIT Delhi

- Anil Kumar Vullikanti, Virginia Tech

- Animesh Mukherjee, IIT Kharagpur

- Anirban Dasgupta, IIT Gandhinagar

- Anoop Cherian, The Australian National University

- Anshuman Dutt, MSR

- Anush Sankaran, IBM Research

- Arijit Biswas, Amazon

- Arnab Bhattacharya, IIT Kanpur

- Arun Rajkumar, Conduent Labs

- Balaji Vasan Srinivasan, Adobe Research

- Balamurali A R, Acalvio

- CV Jawahar, IIIT-Hyderabad

- Debapriyo Majumdar, ISI Kolkata

- Dinesh Garg, IIT Gandhinagar

- Diptikalyan Saha, IBM Research

- Durga Toshniwal, IIT Roorkee

- Ganesh Ramakrishnan, IIT Bombay

- Gautam Shroff, TCS

- Himanshu Bhatt, American Express

- Himanshu Gupta, IBM Research

- Indrajit Bhattacharya, TCS

- Jayadeva, IIT Delhi

- Kamalakar Karlapalem, IIIT-Hyderabad

- Karin Murthy, IBM Research

- Karthik Sankaranarayanan, IBM Research

- Krishna Kummamuru, Accenture

- Krishna Reddy Polepalli, IIIT-Hyderabad

- Lavanya Tekumalla, Amazon

- $\quad$ Liang Hu, UTS

- Lini Thomas, IIIT-Hyderabad

- Maleq Khan, Virginia Tech

- Manish Singh, Microsoft

- Manjira Sinha, Conduent Labs

- Maunendra Sankar Desarkar, IIT Hyderabad

- Medha Atre, IIT Kanpur

- Mitesh M Khapra, IIT Madras

- Narayanan Unny, Conduent Labs

- Niraj Kumar, Phenom People

- Niranjan Balasubramanian, Stony Brook University 
- Niyati Chhaya, Adobe Research

- P Sreenivasa Kumar, IIT Madras

- P.K. Srijith, IIT Hyderabad

- Pabitra Mitra, IIT Kharagpur

- Partha Talukdar, IISc

- Pawan Goyal, IIT Kharagpur

- Piyush Rai, IIT Kanpur

- Pramod Kompalli, Flipkart

- Pratik Jawanpuria, Amazon

- Purushottam Kar, IIT Kanpur

- Ramasuri Narayanam, IBM Research

- Raymond Ng, University of British Columbia

- Ritwik Sinha, Adobe Research

- Rohith D Vallam, IBM Research

- S. Sudarshan, IIT Bombay

- Sakethanath Jagarlapudi, IIT Bombay

- Samarth Bharadwaj, IBM Research

- Sambuddha Roy

- Sandipan Dandapat, Microsoft

- Saptarshi Ghosh, IIT Kharagpur

- Sathya Peri, IIT Hyderabad

- Satyanarayana R Valluri, EPFL

- Senjuti Basu Roy, New Jersey Institute of Technology

- Shantanu Godbole, IBM Research

- Shivali Agarwal, IBM Research

- Snigdha Chaturvedi, UIUC

- Sourangshu Bhattacharya, IIT Kharagpur

- Srikanta Bedathur, IBM Research

- Srinath Srinivasa, IIIT-Bangalore

- Sudeepa Roy, Duke University

- Tanmoy Chakraborty, IIIT-Delhi

- Thomas Lukasiewicz, University of Oxford

- Tirthankar Dasgupta, TCS

- Tushar Khot, University Of Wisconsin-Madison

- Vaibhav Rajan, NUS, Singapore

- Vasudeva Varma, IIIT-Hyderabad

- Vijay Gabale, Huew

- Vikram Goyal, IIIT-Delhi

- Vinay Namboodiri, IIT Kanpur

- Wei Lu, Rupert Labs Inc 


\section{Table of Contents}

\section{Research Track:}

Studying the Influence of Requesters in Posted-price Crowdsourcing.

Malay Bhattacharyya, Indian Institute of Engineering Science and Technology, Shibpur | Sankar Kumar Mridha.

HDSAnalytics: A Data Analytics Framework for Heterogeneous Data Sources.

Yogalakshmi Jayabal | Chandrashekar Ramanathan | Santhanagopalan Rajagopalan, International Institute of Information Technology, Bangalore

A novel topic modeling based weighting framework for class imbalance learning.

Sudarsun Santhiappan | Jeshuren Chelladurai | Ravindran Balaraman, Indian Institute of Technology Madras.

Unsupervised Stance Classification in Online Debates.

Subrata Ghosh | Anand Konjengbam | Sailaja Rajanala | Apparreddy Bharath Reddy | Manish Singh, Indian Institute of Technology, Hyderabad.

Are Saddles good enough for Neural Networks.

Adepu Ravi | Vineeth Balasubramanian, Indian Institute of Technology, Hyderabad.

Comparative Benchmarking of Causal Discovery Algorithms.

Karamjit Singh | Garima Gupta | Vartika Tewari | Gautam Shroff, TATA

CONSULTANCY SERVICES (TCS) Research, Delhi, India.

Fault in your stars: An Analysis of Android App Reviews.

Rahul Aralikatte | Giriprasad Sridhara | Neelamadhav Gantayat | Senthil Mani, IBM.

Potholes and Bad Road Conditions- Mining Twitter to Extract Information on Killer Roads.

Swati Agarwal, BITS Pilani Goa | Nitish Mittal, noon.com | Ashish Sureka, Ashoka University

Online Anomaly Detection with Concept Drift Adaptation using Recurrent Neural Networks.

Sakti Saurav, Indraprastha Institute of Information Technology, Delhi | Pankaj Malhotra | Vishnu Tv | Narendhar Gugulothu | Lovekesh Vig | Puneet Agarwal | Gautam Shroff, TATA CONSULTANCY SERVICES (TCS) LIMITED

Bayesian Optimisation with Prior Reuse for Motion Planning in Robot Soccer. Abhinav Agarwalla | Arnav Kumar Jain | Kv Manohar, Indian Institute of Technology, Kharagpur. 
Product Discovery from E-Commerce Listings via Deep Text Parsing.

Uma Sawant, IITB, LinkedIn | Vijay Gabale, Huew

MineAr: Using Crowd Knowledge for Mining Association Rules in the Health Domain.

Milan Someswar | Arnab Bhattacharya, Indian Institute of Technology, Kanpur

A Neural Attention Based Approach for Clickstream Mining.

Chandramohan T N | Balaraman Ravindran, Indian Institute of Technology, Madras

Efficient RDF Dictionaries with B+ Trees.

Gurkirat Singh | Dhawal Upadhyay | Medha Atre, Indian Institute of Technology, Kanpur

Online Domain Adaptation by Exploiting Labeled Features and Pro-active Learning. Raghu Krishnapuram, Ramaiah Institute of Technology | Arun Rajkumar, Conduent Labs | Adithya Acharya | Nikhil Dhara | Manjunath Goudar | Akshay Sarashetti, Ramaiah Institute of Technology

Syncretic Matching: Story Similarity Between Documents.

Sumant Kulkarni | Srinath Srinivasa | Tahir Dar, International Institute of Information Technology, Bangalore

Supervised Heterogeneous Transfer Learning using Random Forests. Sanatan Sukhija | Narayanan C Krishnan, Indian Institute of Technology Ropar, Punjab, India | Deepak Kumar, Sant Longowal Institute of Engineering and Technology, Longowal, Punjab, India

Finding Shell Company Accounts using Anomaly Detection. Devendra Kumar Luna, Indian Institute of Technology, Kanpur | Girish Palshikar, Tata Research Development and Design Centre | Manoj Apte, Tata Consultancy Services | Arnab Bhattacharya, Indian Institute of Technology, Kanpur

User Bias Removal in Review Score Prediction.

Rahul Wadbude, Indian Institute of Technology, Kanpur | Vivek Gupta, Microsoft |Dheeraj Mekala | Harish Karnick,Indian Institute of Technology, Kanpur

CitiCafe: Conversation-Based Intelligent Platform for Citizen Engagement. Amol Dumrewal, IIT Madras | Anwesh Basu, The University of Texas at Austin | Shubham Atreja | Prateeti Mohapatra | Pooja Aggarwal | Gargi B. Dasgupta, IBM

Scalable linear classifiers based on exponential loss function.

Sandhya Tripathi | Nandyala Hemachandra, Indian Institute of Technology Bombay

Priority Based Functional Group Identification of Organic Molecules using Machine Learning.

Rushikesh Nalla | Rajdeep Pinge | Manish Narwaria |Bhaskar Chaudhury, DA-IICT, Gandhinagar 
STWalk: Learning Trajectory Representations in Temporal Graphs.

Supriya Pandhre, Indian Institute of Technology Hyderabad |Himangi Mittal, Jaypee Institute of Information Technology | Manish Gupta, Microsoft, Hyderabad | Vineeth $\mathrm{N}$ Balasubramanian, Indian Institute of Technology Hyderabad

FuTSe: A Fuzzy Taxonomy Service to Facilitate Product Search.

Prateeti Mohapatra | Anupama Ray | Gargi Dasgupta, IBM Research Lab

Revisiting Tendency based Collaborative Filtering for Personalized Recommendations.

230

Rama Syamala Sreepada | Bidyut Kr. Patra | Avijit Chakrabarty | Satyadev Chandak, National Institute of Technology Rourkela

Some new PAC-Bayesian bounds and their use in selection of regularization parameter for linear SVMs.

Puja Sahu | Nandyala Hemachandra, Indian Institute of Technology Bombay

ADINE: An Adaptive Momentum Method for Stochastic Gradient Descent. Vishwak Srinivasan | Adepu Ravisankar | Vineeth Balasubramanian, Indian Institute of Technology, Hyderabad

Believe It or Not! Identifying Bizarre News in Online News Media.

Vijayasaradhi Indurthi | Subba Reddy Oota | Manish Gupta | Vasudeva Varma, International Institute of Information Technology, Hyderabad

KlusTree: Clustering Answer Trees from Keyword Search on Graphs.

Madhulika Mohanty | Maya Ramanath, Indian Institute of Technology, Delhi

Stance Classification of Multi-Perspective Consumer Health Information. 


\section{Industry Track:}

Mining Entities and their Values from Semi-Structured Documents in Business Process Outsourcing.

Chinnappa Guggilla | Ankit G Pandey | Krishna Kummamuru | Madhura Shivaram, Accenture Solutions Pvt Ltd.

Ensemble Learning for Credit Card Fraud Detection. Ishan Sohony, Pune Institue of Computer Technology, Pune | Rameshwar Pratap, Chennai Mathematical Institute | Ullas Nambiar, Zenlabs, Zensar Technologies, Pune

Anomaly Detection in IloT: A Case Study using Machine Learning. Gauri Shah | Aashis Tiwari, Persistent Systems Ltd.
282

289

295

\section{Young Researchers' Symposium Track:}

\begin{tabular}{|c|c|}
\hline $\begin{array}{l}\text { Identifying consensus block structures in multi-relational network. } \\
\text { Srinka Basu, University of Kalyani| Somshekhar Das | Ujjwal Maulik, Jadavpur } \\
\text { University }\end{array}$ & 301 \\
\hline $\begin{array}{l}\text { Priority Inversion in DRTDBS: Challenges and Resolutions. } \\
\text { Sarvesh Pandey | Udai Shanker, Madan Mohan Malaviya University of Technology }\end{array}$ & 305 \\
\hline $\begin{array}{l}\text { Label Space Driven Feature Space Remapping. } \\
\text { Sanatan Sukhija, IIT Ropar }\end{array}$ & 310 \\
\hline $\begin{array}{l}\text { Is the Cost of Proof-of-Work Consensus Quasilinear? } \\
\text { Aditya Asgaonkar | Pranav Palande | Ramprasad S Joshi, BITS-Pilani }\end{array}$ & 314 \\
\hline $\begin{array}{l}\text { Network based Mechanisms for Competitive Crowdsourcing. } \\
\text { Sankar Kumar Mridha, Indian Institute of Engineering Science and Technology }\end{array}$ & 318 \\
\hline $\begin{array}{l}\text { Handling Cold-start Scenarios in Point-of-Interest Recommendations Through } \\
\text { Crowdsourcing. } \\
\text { Pramit Mazumdar | Bidyut Kr. Patra | Korra Sathya Babu, National Institute of } \\
\text { Technology Rourkela }\end{array}$ & 322 \\
\hline $\begin{array}{l}\text { A Comparative Study of Feature Extraction Methods from User Reviews for } \\
\text { Recommender Systems. } \\
\text { Pradnya Bhagat | Jyoti Pawar, Goa University }\end{array}$ & 325 \\
\hline $\begin{array}{l}\text { Enriching Domain Ontologies using Question-Answer Datasets. } \\
\text { Subhashree S | P Sreenivasa Kumar, Indian Institute of Technology Madras }\end{array}$ & 329 \\
\hline $\begin{array}{l}\text { Online Learning Isotonic Separation for the study of large-scale data. } \\
\text { Ashthika Balakrishnan, PSG College of Technology }\end{array}$ & 333 \\
\hline
\end{tabular}




\begin{tabular}{l|l|}
\hline Deep CNN based Pseudo-concept Selection and Modeling for Generation of Semantic \\
Multinomial Representation of Scene Images. \\
Shikha Gupta | Dileep Aroor Dinesh, IIT Mandi | Veena Thenkanidiyoor, NIT Goa \\
Similarity Search of Time Series Trajectories based on Shape. \\
Gajanan Gawde, Goa College of Engineering | Jyoti Pawar, Goa University \\
Mitotic v/s non-mitotic HEp-2 cells classification for CAD based Auto-Immune \\
$\begin{array}{l}\text { Disorder detection: A study using CNN. } \\
\text { Krati Gupta | Arnav Bhavsar | Anil Sao, Indian Institute of Technology Mandi }\end{array}$ \\
$\begin{array}{l}\text { LAWBO: A Smart Lawyer Chatbot. } \\
\text { Shubhashri Govindarajan | Unnamalai N | Kamalika G, PSG College of Technology }\end{array}$ \\
$\begin{array}{l}\text { A Mobile App based Smoking Cessation Assistance using Automated detection of } \\
\text { Smoking Behavior. } \\
\text { Saurabh Singh Thakur | Ram Babu Roy, IIT Kharagpur }\end{array}$ \\
$\begin{array}{l}\text { Mental health analysis using deep learning for feature extraction. } \\
\text { Deepali Joshi, VIT \& DOT Pune | Mohit Makhija | Yash Nabar, VIT Pune | Dr.Manasi } \\
\text { Patwardhan, TCS Research }\end{array}$ \\
$\begin{array}{l}\text { Feedback Based Real Time Facial and Head Gesture Recognition For e-learning } \\
\text { System. } \\
\text { Shubhada Deshmukh, VIT | Manasi Patwardhan, TCS Research | Anjali Mahajan, PIET }\end{array}$ \\
$\begin{array}{l}\text { Unsupervised Cost Sensitive Predictions with Side Information. } \\
\text { Arun Verma | Manjesh Kumar Hanawal, Indian Institute of Technology Bombay }\end{array}$ \\
\end{tabular}

